\title{
Volume and Enthalpy Relaxation in $\mathrm{Pd}_{42.5} \mathrm{Cu}_{30} \mathrm{Ni}_{7.5} \mathrm{P}_{20}$ Bulk Metallic Glass
}

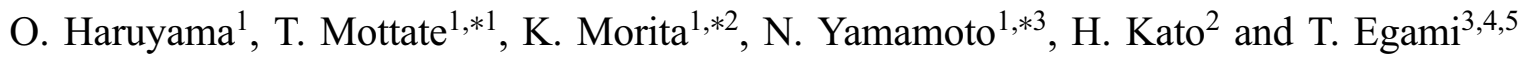 \\ ${ }^{1}$ Department of Physics, Tokyo University of Science, Noda 278-8510, Japan \\ ${ }^{2}$ Institute for Materials Research, Tohoku University, Sendai 980-8577, Japan \\ ${ }^{3}$ Department of Material Science and Engineering, University of Tennessee, Knoxville, Tennessee 37996, USA \\ ${ }^{4}$ Department of Physics and Astronomy, University of Tennessee, Knoxville, Tennessee 37996, USA \\ ${ }^{5}$ Oak Ridge National Laboratory, Oak Ridge, Tennessee 37831, USA
}

\begin{abstract}
The kinetics of structural relaxation in $\mathrm{Pd}_{42.5} \mathrm{Cu}_{30} \mathrm{Ni}_{7.5} \mathrm{P}_{20}$ bulk metallic glass (BMG) was investigated by means of volume relaxation and enthalpy relaxation in the temperature range below $T_{\mathrm{g}}(\approx 573 \mathrm{~K})$. The measured relaxation time was significantly longer than the $\alpha$-relaxation time reported by dynamical mechanical analysis (DMA), indicating that these two relaxation processes are fundamentally different from each other. The temperature dependence of electrical resistivity suggests that the origin of the $\beta$-relaxation process that occurs between room temperature and $T_{\mathrm{g}}$ may be the compositional short range ordering. Anomalous volume expansion was observed in the initial stage of relaxation, which was attributed to annihilation of the $p$-type defects with very short relaxation time. [doi:10.2320/matertrans.MBW201320]
\end{abstract}

(Received October 15, 2013; Accepted November 29, 2013; Published January 18, 2014)

Keywords: bulk metallic glass, structural relaxation, $\beta$ relaxation, volume change, enthalpy change

\section{Introduction}

The structural relaxation in metallic glasses has been studied by static relaxation analysis (SRA) and dynamic mechanical analysis (DMA). The DMA measures the response to dynamic stress fields as a function of frequency and temperature. The $\alpha$-relaxation (main relaxation), which is due to cooperative motion of atoms, is observed typically for a supercooled liquid above the glass transition temperature, $T_{\mathrm{g}}$, whereas the $\beta$-relaxation (secondary relaxation, frequently called Johari-Goldstein $\beta$-relaxation) is observed at temperatures below $T_{\mathrm{g} .}{ }^{1-5)}$ The temperature dependence of the $\alpha$ relaxation is described by Vogel-Fulcher-Tammann (VFT) law, and the $\beta$-relaxation follows Arrhenius law. In the case of insulating glasses, such as polymeric and oxide glasses, the dynamical relaxation phenomena can be observed by dielectric or mechanical loss measurement. However, for metallic glasses the dynamical relaxation can be measured only by mechanical analysis where the shear modulus and the mechanical loss are measured as a function of temperature and frequency. The structural relaxation can be observed by various SRA measurements of a variety of physical properties, such as viscosity, ${ }^{6-11)}$ positron annihilation lifetime, ${ }^{12,13)}$ enthalpy, ${ }^{11,14-16,19,20)}$ and volume ${ }^{11,17-20)}$ as well as mechanical properties. $^{21)}$

Structural relaxation was reported by $\mathrm{Fan}^{14)}$ by means of enthalpy relaxation in a temperature range just below $T_{\mathrm{g}}$ for $\mathrm{Pd}_{43} \mathrm{Cu}_{27} \mathrm{Ni}_{10} \mathrm{P}_{20}$ BMG. The $\alpha$-relaxation in $\mathrm{Pd}_{40} \mathrm{Cu}_{30} \mathrm{Ni}_{10} \mathrm{P}_{20}$ $\mathrm{BMG}$ was investigated by dynamic loss modulus experiments in a temperature range above $T_{\mathrm{g} .}{ }^{3,5)}$ Recently, the kinetics of the dynamic $\alpha$-relaxation below $T_{\mathrm{g}}$ in $\mathrm{Pd}_{42.5} \mathrm{Cu}_{30^{-}}$ $\mathrm{Ni}_{7.5} \mathrm{P}_{20}$ BMG was studied by Kato. ${ }^{22}$ ) In this case, the kinetics of resonant frequency was described by Arrhenius equation with a pre-factor of $2.0 \times 10^{-69} \mathrm{~s}$ and activation

\footnotetext{
${ }^{* 1}$ Graduate Student, Tokyo University of Science. Present address: Fujitsu Mission Critical Systems Ltd., Yokohama 221-0013, Japan

${ }^{* 2}$ Graduate Student, Tokyo University of Science. Present address: TDKEPC Co. Ltd., Honjyo 015-0062, Japan

${ }^{* 3}$ Graduate Student, Tokyo University of Science
}

energy of $7.96 \mathrm{eV}$. The evolution of different types of $\beta$ relaxation has been reported by DMA in a temperature range below $T_{\mathrm{g}}{ }^{5,22,23)}$ For instance, they observed the $\beta$-relaxation events $^{23)}$ related to hopping diffusion of $\mathrm{P}$ atom with a very short relaxation time of approximately $0.01 \mathrm{~s}$ at $512 \mathrm{~K}$, about $60 \mathrm{~K}$ below $T_{\mathrm{g}}$.

A problem with the static measurements is that it is impossible to access the processes with the relaxation time shorter than approximately $10 \mathrm{~s}$ or so, which makes it impossible to measure the $\alpha$-relaxation in a temperature region above $T_{\mathrm{g}}$ and the $\beta$-relaxation with a short relaxation time, about less than a few seconds. Thus the two methods, SRA and DMA, have little overlap, making it difficult to construct a total picture of the relaxation mechanisms in the supercooled liquid and glassy solid regions. In the present study, we examined the structural relaxation over a temperature range just below $T_{\mathrm{g}}$ by means of both volume relaxation and enthalpy relaxation, covering a wider temperature range than the previous study. ${ }^{14)}$ The results were then compared with the $\alpha$-relaxation measured by DMA. In addition the evolution of the temperature dependence of electrical resistivity from room temperature to $T_{\mathrm{g}}$ was studied, which suggests that the origin of the $\beta$-relaxation in this sample may be the compositional short-range ordering.

\section{Experimental}

The master alloy ingot with a composition of $\mathrm{Pd}_{42.5^{-}}$ $\mathrm{Cu}_{30} \mathrm{Ni}_{7.5} \mathrm{P}_{20}$ was prepared by sintering method, when the mixture of pure elements $(99.5 \% \mathrm{Pd}, 99.99 \% \mathrm{Cu}, 99.99 \% \mathrm{Ni}$, $99.99995 \% \mathrm{P}$ ) was melt at $1473 \mathrm{~K}$ for $12 \mathrm{~h}$ in an evacuated quartz tube. Next, the alloy sintered was repeatedly melted and held at $1473 \mathrm{~K}$ for $12 \mathrm{~h}$ to homogenize the alloy composition. Prior to the vitrification of the melt, it was flux-treated with a dehydrated $\mathrm{B}_{2} \mathrm{O}_{3}$ at $1300 \mathrm{~K}$ for $12 \mathrm{~h}$ to remove phosphorous oxides formed during preparing the master alloy. Bulk metallic glass (BMG) was obtained by water-quenching the melt of master alloy with a size of $8 \mathrm{~mm}$ in diameter and $40 \mathrm{~mm}$ in length. The detail of sample 
preparation is published everywhere. ${ }^{18-20)}$ The composition of quenched BMG was investigated by the induced coupling plasma technique, and it was within \pm 0.2 at $\%$ for each nominal composition. The characterization of glass structure was performed by $\mathrm{X}$-ray diffraction with $\mathrm{Cu}-K_{\alpha}$ radiation and differential thermal analysis (DTA), and it was verified that the sample was composed of a single amorphous phase. The volume of the sample was determined from the mass density measured at room temperature by Archimedes method with a Mettler Toledo AX26 electro-balance and $n$-Tridecane as a working fluid. The enthalpy change of the sample was calculated by integrating the specific heat curve with temperature. The specific heat curve was measured with a Perkin Elmer Pyris 1 differential scanning calorimeter (DSC) at a heating rate of $0.167 \mathrm{~K} / \mathrm{s}$ under atmospheric pressure. To sustain a good precision in the specific heat measurement, it is desirable to use a smaller rate. We determined a heating rate of $0.167 \mathrm{~K} / \mathrm{s}$ after testing measurement under some different rates. Then the sample weight was controlled within $99 \pm 0.05 \mathrm{mg}$. The heat flow of the sample plus an aluminum (Al) pan, the heat flows of standard sapphire (a weight of $128.95 \mathrm{mg}$ ) plus the $\mathrm{Al}$ pan and only the $\mathrm{Al}$ pan were subsequently measured. Three heat flow experiments mentioned above were treated as a single set, and multi-sets of heat flow experiments were repeated to construct an enthalpy relaxation curve. The specific heat of the sample was obtained by correcting the heat flow of the sample with the heat flow and specific heat of the standard sapphire. To examine the structural relaxation over a wide temperature range, the electrical resistance of the sample was measured at room temperature after accomplishing each isochronal annealing operation with a temperature step of $20 \mathrm{~K}$ and duration of $600 \mathrm{~s}$ up to $T_{\mathrm{g}}$. The sample size was approximately $1.5 \times 1.5 \times 15 \mathrm{~mm}^{3}$. For the electrical resistivity measurement the DC four-probe technique was used with a Keithley 2182 nano-voltmeter and 2400 current source meter. In the present study, the annealing operation of the sample was carefully conducted because inappropriate heat treatment led to ambiguous interpretation for volume and enthalpy variation during relaxation. The enthalpy relaxation was investigated by measuring the enthalpy recovery of relaxed BMG samples, for which they were annealed in a pre-heated silicon oil bath instead of in a DSC apparatus. The volume relaxation was studied by the change in mass density of relaxed BMG samples at room temperature, when they were sequentially annealed at a given temperature in a silicon oil bath. The annealed BMGs were cooled down to room temperature as quickly as possible after accomplishing a given heat treatment. In the case of electrical resistance measurements, the sample was annealed in an ULVAC RHLE410 infrared furnace system with a pure Ar gas to achieve required fast heating and cooling operations.

\section{Results}

\subsection{Characterization of the as-quenched BMG}

The amorphous nature and thermodynamic property are examined by X-ray diffraction and DTA conducted with a heating rate of $0.33 \mathrm{~K} / \mathrm{s}$. The results are shown in Fig. 1(a) X-ray diffraction and (b) DTA thermogram. No Bragg peaks
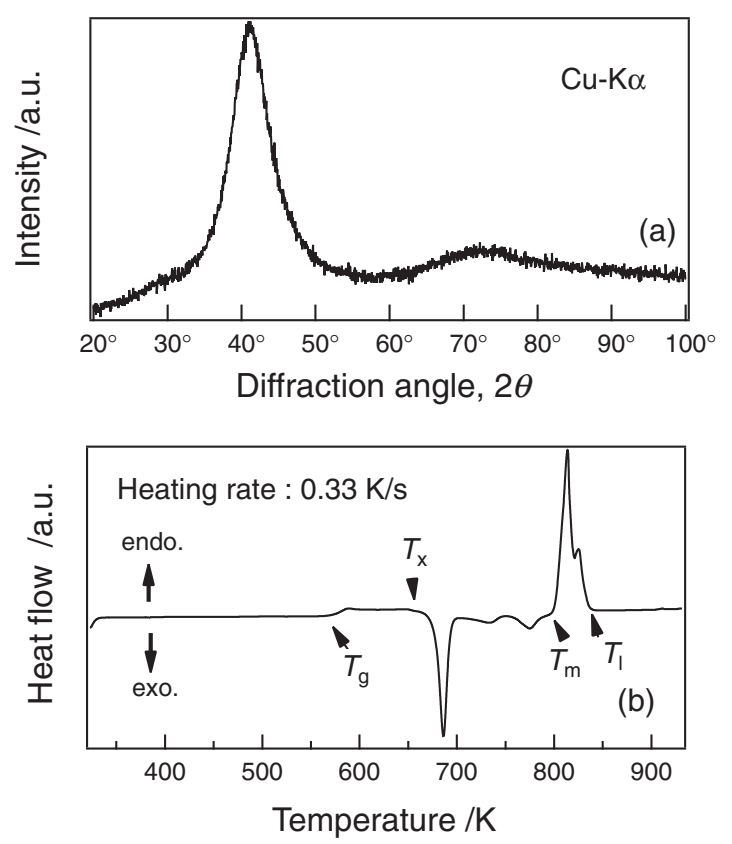

Fig. 1 (a) X-ray diffraction of as-quenched BMG measured with a $\mathrm{Cu}-\mathrm{K} \alpha$ radiation, and (b) DTA thermogram taken with a heating rate of $0.33 \mathrm{~K} / \mathrm{s}$.

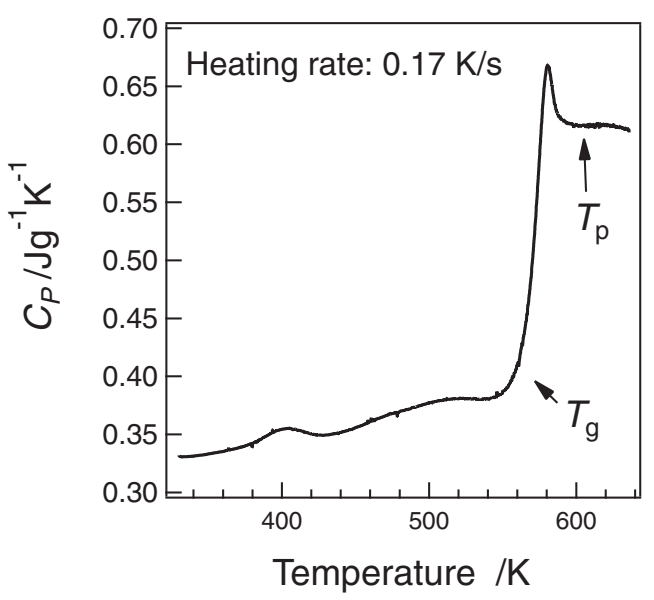

Fig. 2 The specific heat curve of as-quenched BMG measured with a heating rate of $0.17 \mathrm{~K} / \mathrm{s}$. Valleys around 420 and $550 \mathrm{~K}$ are due to the heat release accompanied by structural relaxation, while the peak at $590 \mathrm{~K}$ is due to the heat absorption in glass transition. $T_{\mathrm{g}}(=573 \mathrm{~K}$ at a rate of $0.33 \mathrm{~K} / \mathrm{s})$ and pre-annealing temperature, $T_{\mathrm{p}}(=613 \mathrm{~K})$, are shown by arrows.

due to any crystalline phases can be observed in the diffraction pattern, and the glass transition temperature, $T_{\mathrm{g}}=573 \mathrm{~K}$, the crystallization temperature, $T_{\mathrm{x}}=662 \mathrm{~K}$, the melting temperature, $T_{\mathrm{m}}=800 \mathrm{~K}$, and the liquidus temperature, $T_{1}=833 \mathrm{~K}$, determined by DTA are indicated in Fig. 1(b). Figure 2 shows the specific heat curve of asquenched BMG. A rapid increase in specific heat is observed in the glass transition, and it is followed by evolution of endothermic peak. Prior to the glass transition, two exothermic peaks can be observed, one near $T_{\mathrm{g}}$ and another at low temperatures around $420 \mathrm{~K}$. The high temperature exothermic peak can be identified with the $\alpha$-relaxation. The low temperature exothermic peak is probably ascribed to the chemical short range ordering (CSRO) process. ${ }^{24,25)}$ Such evolution of exothermic peak was frequently observed in 


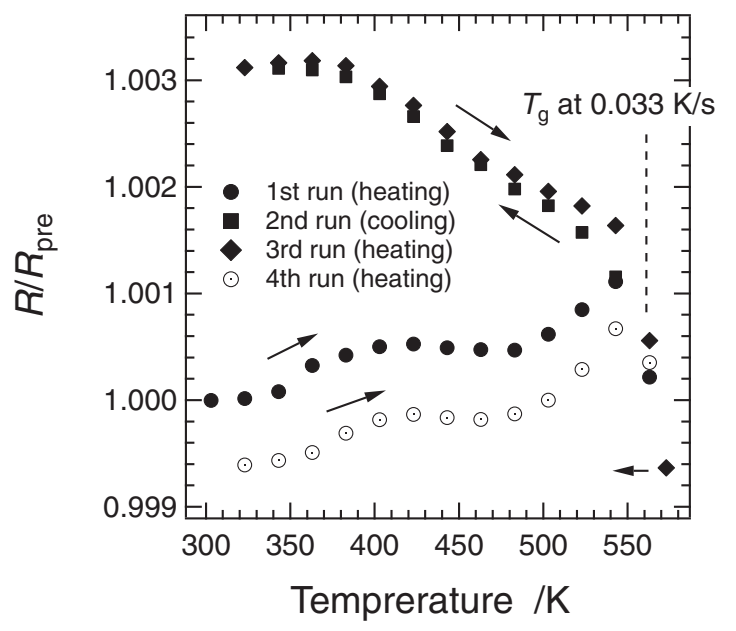

Fig. 3 The change in electrical resistance, $R / R_{\text {pre, }}$ during isochronal annealing is plotted against temperature, where $R$ and $R_{\text {pre }}$ are electrical resistances of relaxed sample and pre-annealed one, respectively. The annealing operation was repeated between room temperature and $T_{\mathrm{g}} \approx 560 \mathrm{~K}$ that was lowered due to used small average heating rate of $0.033 \mathrm{~K} / \mathrm{s}$.

amorphous alloys ${ }^{26-28)}$ prepared by melt-spinning, and its origin has been understood as the evolution of CSRO process at low temperatures and structural relaxation near $T_{\mathrm{g}}$.

\subsection{Resistivity and structural relaxation below $T_{\mathrm{g}}$}

The variation in electrical resistance during thermal scan with a heating rate of $0.033 \mathrm{~K} / \mathrm{s}$ is shown in Fig. 3. The sample was pre-annealed at $613 \mathrm{~K}$ in the supercooled liquid region for $300 \mathrm{~s}$ in order to reduce inhomogeneity in the fictive temperature, or the degree of structural relaxation, in the as-quenched BMG. For this reason all relaxation experiments were carried out after pre-annealing. In the present study, mass density was measured by using relatively huge BMGs with a weight of about $2 \mathrm{~g}$ and a size of $8 \mathrm{~mm}$ in diameter and $4 \mathrm{~mm}$ in length. Firstly the heating curve was measured from room temperature up to $T_{\mathrm{g}}$. In this run $T_{\mathrm{g}}$ is shifted to a lower value, about $560 \mathrm{~K}$, than that $(573 \mathrm{~K})$ determined by DTA. This must be due to the difference in the heating rate, $0.33 \mathrm{~K} / \mathrm{s}$ for DTA and $0.033 \mathrm{~K} / \mathrm{s}$ for resistivity measurement. Two peaks appear in the 1 st heating curve; the broad peak at low temperatures is in good agreement in position with that of the specific heat curve of the asquenched BMG. Subsequently the cooling curve was measured from $T_{\mathrm{g}}$ down to room temperature at the rate of $-0.033 \mathrm{~K} / \mathrm{s}$ (the 2 nd run). As shown in Fig. 3 the electrical resistance increases with decreasing temperature and reaches almost a constant value around room temperature. Apparently the structural relaxation occurred during the first heating cycle resulted in the increased resistivity at room temperature. In other words, the decrease of fictive or effective temperatures results in the increase of resistivity at room temperature in this sample. After that, the 3rd run (second heating run) was made again up to $T_{\mathrm{g}}$. We found that the electrical resistance decreased with increasing temperature almost along the cooling curve. The structure is already relaxed during the first run, so the second heating did not bring about any additional relaxation. However, it is interesting to note that the second heating was continued up to $573 \mathrm{~K}$, about $10 \mathrm{~K}$ higher than $T_{\mathrm{g}}$. As shown in Fig. 3, the resistance values at $566 \mathrm{~K}$ and at $573 \mathrm{~K}$, both above $T_{\mathrm{g}}$, are lower than the extrapolation from the data below $T_{\mathrm{g}}$. This is because by bringing the sample to temperatures above $T_{\mathrm{g}}$ the structural relaxation induced by the first run was undone, and the sample was rejuvenated. By rapidly cooling the sample to room temperature we confirmed that the sample last relaxed at $573 \mathrm{~K}$ has the room temperature resistivity even lower than the one for the first run. This indicates that the fictive temperature of the pre-annealed BMG is below $573 \mathrm{~K}$ and slightly above $T_{\mathrm{g}}$. Even though the sample was pre-annealed at $613 \mathrm{~K}$ the sample apparently kept relaxing during cooling after pre-annealing down to a temperature close to $T_{\mathrm{g}}$. We then measured the 4 th run (third heating run) up to $T_{\mathrm{g}}$. The obtained electrical resistance curve is quite similar to the first heating curve except for a shift to lower values, because the fictive temperature of this sample, $573 \mathrm{~K}$, is lower than that of the as-quenched sample.

It is interesting to note that there is a broad peak around $420 \mathrm{~K}$ in the first run which shifts to about $360 \mathrm{~K}$ in the second and third run. This peak comes back to $420 \mathrm{~K}$ in the 4 th run. The reversible nature of this peak in the resistance measurements and the broad exothermic peak in the specific heat curve suggest that this peak is due to the compositional short-range ordering (CSRO) process. Dynamic mechanical analysis (DMA) also reported a broad loss peak around $420 \mathrm{~K}$, which was identified as Johari-Goldstein (JG) $\beta$ relaxation. ${ }^{3,5)}$ Therefore we conjecture that the origin of the $\mathrm{JG} \beta$-relaxation in dynamic relaxation is the reversible CSRO process.

\subsection{Structural relaxation in a $\mathbf{P d}_{42.5} \mathrm{Cu}_{30} \mathrm{Ni}_{7.5} \mathbf{P}_{20} \mathrm{BMG}$ 3.3.1 Volume relaxation}

Pre-annealed BMGs were isothermally annealed at various temperatures for a given time and quenched down to room temperature. The mass density was measured at room temperature after each step of annealing and quench-down. The relative volume change, $\Delta V / V_{\text {pre }}=\left\{V(T, t)-V_{\text {pre }}\right\} /$ $V_{\text {pre, }}$, where $V(T, t)$ and $V_{\text {pre }}$ are the specific volume of BMG relaxed at temperature $T$ for time $t$ and that of the preannealed BMG, respectively, were determined from the measured mass densities of BMGs. The results are shown in Fig. 4, where the initial behavior of relaxation at $540 \mathrm{~K}$ is shown in the inset. At first the volume rapidly increases during the relaxation for the initial $120 \mathrm{~s}$, and then decreases monotonically. We confirmed that such volume expansion occurs similarly for as-cast $\mathrm{Pd}_{42.5} \mathrm{Cu}_{30} \mathrm{Ni}_{7.5} \mathrm{P}_{20} \mathrm{BMG}$, as-cast and pre-annealed $\mathrm{Pd}_{43} \mathrm{Cu}_{27} \mathrm{Ni}_{10} \mathrm{P}_{20}$ BMGs. However, other $\mathrm{Pd}-\mathrm{P}$ based BMGs such as $\mathrm{Pd}_{40} \mathrm{Ni}_{40} \mathrm{P}_{20},{ }^{18)} \mathrm{Pd}_{46} \mathrm{Cu}_{35.5} \mathrm{P}_{18.5},{ }^{29)}$ $\mathrm{Pt}_{60} \mathrm{Ni}_{15} \mathrm{P}_{25},{ }^{20)}$ and $\mathrm{Pt}_{40} \mathrm{Cu}_{20} \mathrm{Ni}_{10} \mathrm{P}_{20}{ }^{30)}$ did not exhibit such volume expansion at the initial stage of relaxation. Accordingly, the initial volume expansion is specific to $\mathrm{Pd}-\mathrm{Cu}-\mathrm{Ni}-$ $\mathrm{P}_{20}$ BMGs, which may arise from the peculiarity of the glass structure of the alloy. This effect resembles the Kovacs effect, ${ }^{31)}$ well-known to occur in polymeric and oxide glasses. This point is discussed later.

\subsubsection{Enthalpy relaxation}

The specific heat curve was measured for BMGs relaxed under a variety of conditions. A portion of the curves near $T_{\mathrm{g}}$ are shown in Fig. 5. The heat absorption peak appearing 
in the glass transition is larger for more relaxed BMG, indicating that the enthalpy disappeared during relaxation is recovered in the glass transition by absorbing the heat from the environment. Assuming that a state in the supercooled liquid region is independent of any thermal history, the reduction of enthalpy, $\Delta H_{\mathrm{g}}(T, t)$, of relaxed BMGs during relaxation is expressed as,

$$
\begin{gathered}
\Delta H_{\mathrm{g}}(T, t)=H_{\mathrm{g}}(T, t)-\left.H_{\mathrm{g}, \mathrm{pre}}\right|_{T} \\
\left.H_{\text {SL.pre }}\right|_{615}-\left.H_{\mathrm{g} . p r e}\right|_{T}=\int_{T}^{615} C_{\text {P.pre }}\left(T^{\prime}\right) \mathrm{d} T^{\prime} \\
\left.H_{\mathrm{SL}}(T, t)\right|_{615}-\left.H_{\mathrm{g}}(T, t)\right|_{T}=\int_{T}^{615} C_{\mathrm{P}}\left(T^{\prime}, T, t\right) \mathrm{d} T^{\prime}
\end{gathered}
$$

where $H_{\mathrm{g}}(T, t)$ and $\left.H_{\mathrm{g}, \mathrm{pre}}\right|_{T}$ are enthalpies at $T$ for BMG relaxed at $T$ for $t$, and that of the pre-annealed BMG, respectively. Assuming that enthalpies at $615 \mathrm{~K}$ in the supercooled liquid region, $\left.H_{\mathrm{SL}, p r e}\right|_{615}$ and $\left.\mathrm{H}_{\mathrm{SL}}(T, t)\right|_{615}$, are the same, the eq. (1) can be derived by subtracting eq. (3) from eq. (2). The results are shown in Fig. 6. The enthalpy of the pre-annealed BMG decreases with increasing time, and its change is larger at lower temperatures as expected.

\subsubsection{Kinetics of volume and enthalpy relaxation}

The relaxation data were fitted to the stretched exponential relaxation function, $\Delta P=(\Delta P)_{\mathrm{eq}}\left\{1-\exp \left[-(t / \tau)^{\beta}\right]\right\}$, where $\Delta P$ exhibits the change in physical property during relaxation, and $(\Delta P)_{\mathrm{eq}}$ is the saturation value when $t$ approach infinity. The relaxation time, $\tau$, and Kohlrausch index, $\beta$, were obtained by fitting, and are shown in Figs. 7(a) and 7(b), respectively, as a function of annealing temperature. We also include the results of similar thermal measurement in Ref. 14), which show good agreement with our results. The relaxation times are usually fitted to Vogel-Fulcher-Tammann (VFT) equation in the region near $T_{\mathrm{g}}$. In the present case, both volume and enthalpy relaxation curves bend upward at around $550 \mathrm{~K}$. Such behavior does not obviously allow us to fit each curve to a single function. Consequently, we did not fit relaxation times to VFT equation. Instead, we used the Arrhenius function as approximately expressing all data that include volume relaxation curve and two enthalpy relaxation curves. As will be mentioned later, the pre-factor and activation energy of Arrhenius function gives us important clues with regard to the collective motion of atoms in structural relaxation. Kohlrausch index exhibits a strong temperature dependence, which ranges from about 0.4 at low temperatures to 0.8 or more at high temperatures close to $T_{\mathrm{g}}$. Such strong temperature dependence may have resulted from the closeness of the pre-annealing temperature to the measurement temperature. Indeed the as-cast Zr-based BMGs exhibit weak temperature dependence of Kohlrausch index, ${ }^{32}$ ) which is similar to the result reported by DMA. ${ }^{33)}$

\section{Discussion}

\subsection{Distinction between the relaxation times for SRA and DMA}

The relaxation time, $\tau$, of the structural relaxation is plotted as a function of inverse temperature in Fig. 8. It is compared with the $\alpha$-relaxation time ${ }^{3,5,14,22)}$ determined by the DMA measurement which is conventionally assigned to the structural relaxation in a liquid as a result of cooperative motion of atoms. The DMA relaxation time of supercooled liquid $^{3)}$ (shown by blue squares) is consistent with the extrapolation of the DMA data in the glassy solid region ${ }^{22}$ ) (blue circles). However, the structural relaxation time (black circles, diamonds and triangles) is significantly longer than the DMA relaxation time, particularly in the region close to $T_{\mathrm{g}}$. This result indicates that the structural relaxation in SRA and the $\alpha$-relaxation in DMA are two distinct events. In terms of defect dynamics the $\alpha$-relaxation is caused by the movement of defects, whereas the structural relaxation is caused by annihilation of defects. For instance in the freevolume theory these two events have different time-scales. ${ }^{34)}$ In the shear-transformation-zone (STZ) theory the $\alpha$ relaxation is caused by the activation of the STZ, whereas the structural relaxation is caused by the change in the STZ population, thus the change in the effective temperature. ${ }^{35)}$ In terms of the atomic-level stresses ${ }^{36}$ ) the structural relaxation is the result of annihilation of $p$ and $n$ defects. ${ }^{37,38)}$ The fact that the two time-scales are different supports the view that some minority atoms are responsible for the relaxation phenomena, and justifies the defect model. In computer simulations as well the multi-site relaxation time which is sensitive to dynamic heterogeneity becomes longer than the $\alpha$-relaxation time in supercooled liquids. ${ }^{40)}$

The Arrhenian analysis of the static and dynamic data in the glassy solid region in Fig. 8, where the static data include the enthalpy relaxation data reported by Ref. 14), yielded the relaxation time, $\tau=\tau_{0} \exp \left(E / k_{\mathrm{B}} T\right)$, where pre-factor $\tau_{0}$ is $2.3 \times 10^{-23} \mathrm{~s}$, activation energy $E$ is $2.84 \mathrm{eV}$, and $k_{\mathrm{B}}$ is the Boltzmann constant. These are reasonable values, although the pre-factor is a little too short. A similar analysis of the $\alpha$ relaxation data ${ }^{22)}$ below $T_{\mathrm{g}}$ resulted in unphysical values of $\tau_{0 \alpha}=2.0 \times 10^{-69} \mathrm{~s}$ and $E_{\alpha}$ of $7.98 \mathrm{eV}$. These values reflect the fragile nature of this liquid, and indicate that the structure significantly changes with temperature so that activation energy is temperature dependent, ${ }^{41)}$ and the regular Arrhenian analysis is not applicable. The Maxwell relaxation time is given by $\tau_{\mathrm{M}}=\eta / G_{\infty}$, where $\eta$ is viscosity and $G_{\infty}$ is the high-frequency shear modulus, which is of the order of $10^{10} \mathrm{GPa}$. Thus the Maxwell relaxation time at $T_{\mathrm{g}}$ is of the order of $\tau_{\mathrm{M}}\left(T_{\mathrm{g}}\right)=10^{2} \mathrm{~s}$. If we assume $\tau_{\alpha}=\tau_{\mathrm{M}}$, we obtain $\tau_{\alpha}\left(T_{\mathrm{g}}\right) / \tau_{0 \alpha}=10^{71}$. Then the fragility index, ${ }^{42)} \mathrm{m}=\mathrm{d} \log \eta /$ $\left.\mathrm{d}\left(T_{\mathrm{g}} / T\right)\right|_{T=T_{\mathrm{g}}}$, estimated from this result by neglecting the temperature dependence of $G_{\infty}$ is 71 , slightly higher than the earlier estimate of $58.5^{10)}$

\subsection{Two-defect model}

The volume of BMG became expanded rather than compressed at the initial stage of relaxation, after annealing for $120 \mathrm{~s}$ as shown in Fig. 4. Such a reverse relaxation phenomenon occurs in a partially relaxed sample, and is known as the Kovacs effect. ${ }^{43}$ ) As discussed above even though the sample was pre-annealed at $613 \mathrm{~K}$ the sample relaxed during cooling, resulting in the fictive temperature close to $T_{\mathrm{g}}$. Therefore the fictive temperature may not have been uniform in the sample. It is possible that fast processes with low activation energies reached the fictive temperature lower than $T_{\mathrm{g}}$, and upon annealing resulted in volume expansion. However, if that is the case the amount of volume 


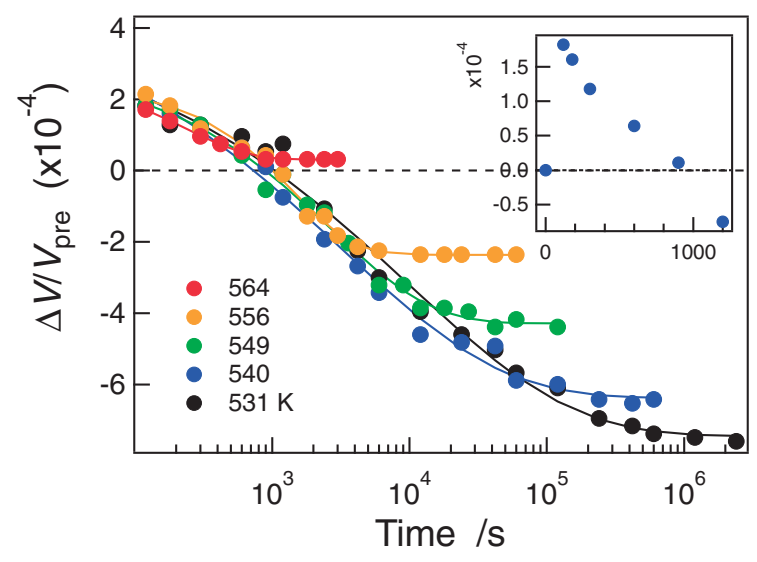

Fig. 4 The isothermal change in the volume of pre-annealed BMG during structural relaxation, where the initial stage of relaxation at $540 \mathrm{~K}$ is enlarged in the inset. Except first point (annealing for $120 \mathrm{~s}$ ) in each running curve, the data points were fitted to the stretched exponential relaxation function.

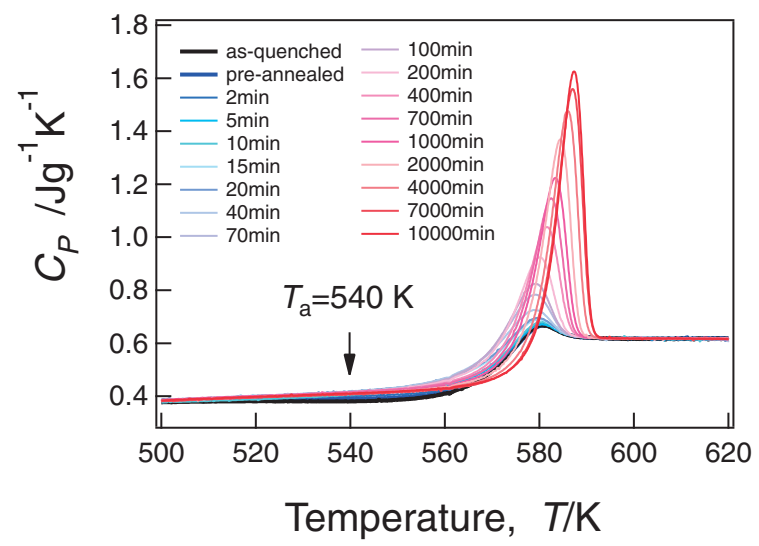

Fig. 5 Specific heat curves of pre-annealed BMGs that were relaxed at $540 \mathrm{~K}$ for a variety of times. The longer relaxed sample shows the larger heat absorption peak to recovery disappeared enthalpy in glass transition.

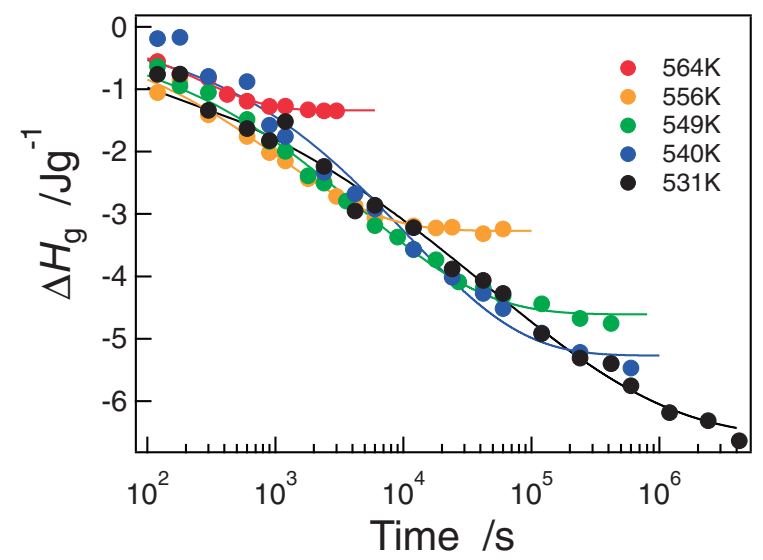

Fig. 6 Enthalpy relaxation curves of pre-annealed BMGs obtained for various annealing temperatures, where each curve was well described by the stretched exponential relaxation function.
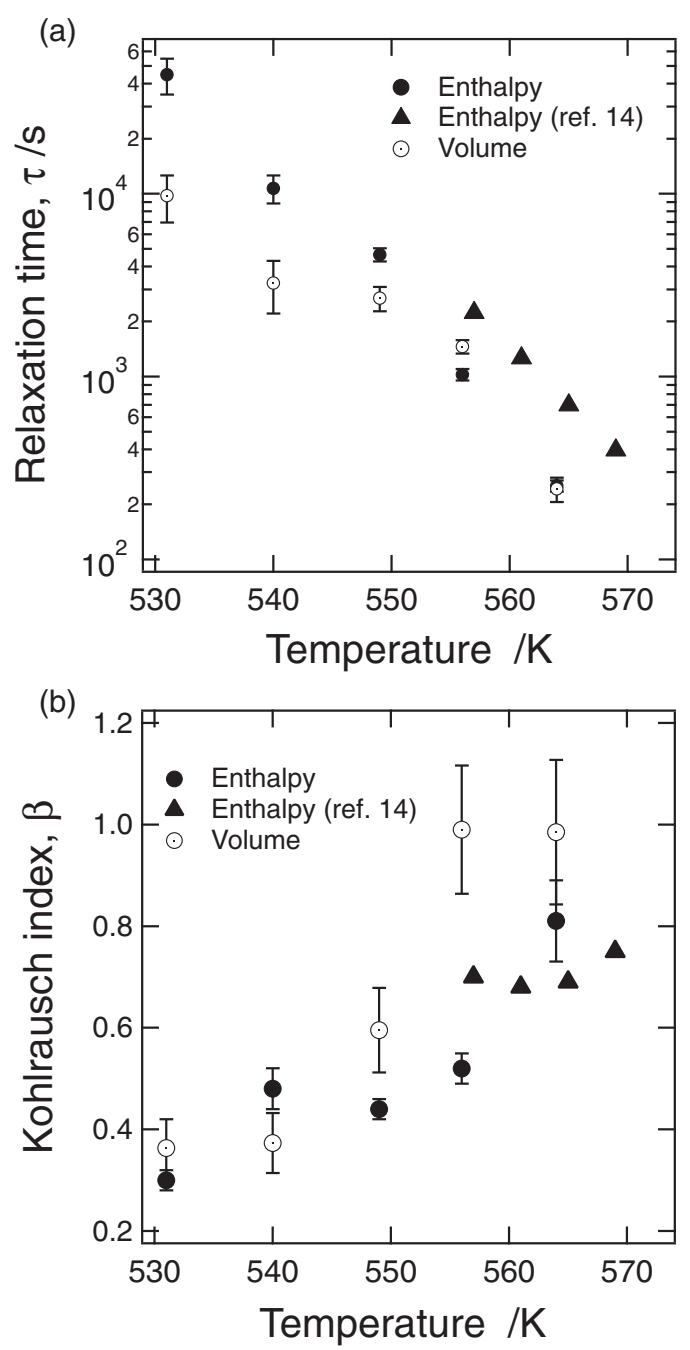

Fig. 7 (a) The relaxation time, and (b) Kohlrausch index were plotted as a function of temperatures, where the enthalpy data include the results $(\boldsymbol{A})$ of Ref. 14).

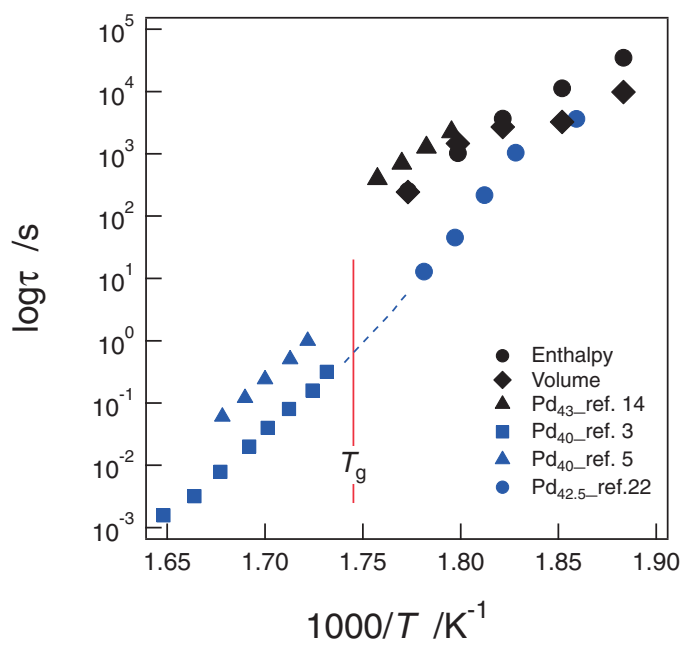

Fig. 8 Relaxation times $\tau$ for static and dynamic relaxations are plotted against $1000 / T$, where dynamic data (blue symbols) include relaxation times measured in both supercooled liquid ${ }^{3,5}$ ) (solid triangles and rectangles) and glassy solid regions ${ }^{22}$ (solid circles). The black symbols denote static data, where the triangles represent static enthalpy data reported. ${ }^{14)}$ The broken line (blue) connecting two DMA data in both regions below and above $T_{\mathrm{g}}$ is drawn as a guide of eyes. 
expansion after $120 \mathrm{~s}$ should depend upon the annealing temperature; the amount of expansion should be larger for higher annealing temperature. Because the amount of volume increase is almost independent of the annealing temperature, we conjecture that the initial volume expansion is not due to the Kovacs effect.

We reported that the P-based metallic glass, typically like $\mathrm{Pt}_{60} \mathrm{Ni}_{15} \mathrm{P}_{25}$, exhibits two-step volume decrement during static relaxation. ${ }^{20)}$ The results were interpreted in terms of the two-defect model for glass structure. ${ }^{37,38)}$ In this model, the atomic-level fluctuation of mass density in the metallic glass is expressed by the atomic volume strain $\varepsilon_{\mathrm{v}}$. The $n$-type and $p$-type defects are defined as the atomic site expanded and compressed, respectively, with the volume strain larger than $11 \% .{ }^{39)}$ Accordingly the volume change during relaxation is induced by the competing processes of two different volume contributions in which annihilation of $n$-type defects decreases its volume on disappearing while annihilation of $p$-type defects oppositely expands the volume. ${ }^{20)}$ The volume changes due to the two processes do not fully cancel each other because of anharmonicity in the interatomic potential, ${ }^{37)}$ resulting in the reduction in the total volume. ${ }^{20)}$ The initial volume expansion observed in the present alloy may be due to some $p$-type defects with very short relaxation times within the two-defect model.

It should be noted that the sign reversal was not observed for enthalpy relaxation, and $\Delta H$ is negative from the start as shown in Fig. 6. This is also consistent with the sign reversal not being due to the Kovacs effect but being due to the competing $p$ - and $n$-type defects. For volume relaxation $p$ and $n$-type defects compete and the effects partially cancel each other. For enthalpy relaxation, on the other hand, they both contribute to reduce $\Delta H$. Thus the two-defect model is more consistent with our results than the Kovacs effect.

\section{Conclusion}

The kinetics of the structural relaxation in $\mathrm{Pd}_{42.5} \mathrm{Cu}_{30^{-}}$ $\mathrm{Ni}_{7.5} \mathrm{P}_{20}$ BMG was investigated by volume and enthalpy relaxation in a glassy solid region below $T_{\mathrm{g}}$. The relaxation process was well described by a stretched exponential relaxation function except for an initial stage of volume relaxation, where small volume expansion was observed rather than the volume reduction. The origin of volume expansion was assigned to the $p$-type defects with a very short relaxation time. The relaxation time of volume and enthalpy relaxation in the present study was much larger than the $\alpha$-relaxation time of the dynamic measurement in a glassy solid region below $T_{\mathrm{g}}$. This result is consistent with the idea that some structural defects are responsible for structural and mechanical relaxation phenomena. The kinetics of volume relaxation is consistent with the two-defect model. The results of the measurement of electrical resistance during relaxation below $T_{\mathrm{g}}$ suggests the existence of the $\beta$-relaxation around $420 \mathrm{~K}$ which may be due to the CSRO.

\section{Acknowledgment}

The work by TE was supported by the US Department of Energy, Office of Basic Energy Sciences, Materials Science and Engineering Division. We also would devote great gratitude to Cooperative Research and Development Center for Advanced Materials, IMR, Tohoku University for supporting the present work.

\section{REFERENCES}

1) H. Okumura, H. S. Chen, A. Inoue and T. Masumoto: Jpn. J. Appl. Phys. 30 (1991) 2553-2557.

2) D. N. Perera and A. P. Tsai: J. Phys. D Appl. Phys. 32 (1999) $2933-$ 2941.

3) Z. F. Zhao, P. Wen, C. H. Shek and W. H. Wang: Phys. Rev. B 75 (2007) 174201.

4) J. C. Qiao and J. M. Pelletier: J. Appl. Phys. 112 (2012) 033518.

5) H. B. Yu, W. H. Wang and K. Samwer: Mater. Today 16 (2013) 183191.

6) E. Bakke, R. Busch and W. L. Johnson: Appl. Phys. Lett. 67 (1995) 3260-3262.

7) A. Masuhr, T. A. Waniuk, R. Busch and W. L. Johnson: Phys. Rev. Lett. 82 (1999) 2290-2293.

8) G. J. Fan, H.-J. Fecht and E. J. Lavernia: Appl. Phys. Lett. 84 (2004) 487-489.

9) T. Yamasaki, S. Maeda, Y. Yokoyama, D. Okai, T. Fukami, H. M. Kimura and A. Inoue: Mater. Trans. 46 (2005) 2746-2750.

10) H. Kato, T. Wada, M. Hasegawa, J. Saida, A. Inoue and H. S. Chen: Scr. Mater. 54 (2006) 2023-2027.

11) Z. Evenson and R. Busch: Acta Mater. 59 (2011) 4404-4415.

12) C. Nagel, K. Rätzke, E. Schmidtke and J. Wolff: Phys. Rev. B 57 (1998) 10224.

13) J. F. Wang, L. Liu, J. Z. Xiao, T. Zhang, B. Y Wang, C. L. Zhou and W. Long: J. Phys. D Appl. Phys. 38 (2005) 946-949.

14) G. J. Fan, J. F. Löeffler, R. K. Wunderlich and H.-J. Fecht: Acta Mater. 52 (2004) 667-674.

15) I. Gallino, M. B. Shah and R. Busch: Acta Mater. 55 (2007) 13671376.

16) M. E. Launey, J. J. Kruzic, C. Li and R. Busch: Appl. Phys. Lett. 91 (2007) 051913.

17) U. Harms, O. Jin and R. B. Schwarz: J. Non-Cryst. Solids 317 (2003) 200-205.

18) O. Haruyama and A. Inoue: Appl. Phys. Lett. 88 (2006) 131906

19) O. Haruyama, Y. Nakayama, R. Wada, H. Tokunaga, J. Okada, T. Ishikawa and Y. Yokoyama: Acta Mater. 58 (2010) 1829-1836.

20) M. Kohda, O. Haruyama, T. Ohkubo and T. Egami: Phys. Rev. B 81 (2010) 092203.

21) M. Weiss, M. Moske and K. Samwer: Appl. Phys. Lett. 69 (1996) 3200-3202.

22) H. Kato, T. Ichitsubo, H. Wang and T. Wada: J. Jpn. Soc. Powder Powder Metall. 60 (2013) 228-235 (in Japanese).

23) X.-P. Tang, J. F. Löffler, R. B. Schwarz, W. L. Johnson and Y. Wu: Appl. Phys. Lett. 86 (2005) 072104.

24) T. Egami: Ann. N. Y. Acad. Sci. 37 (1981) 238-251.

25) M. R. Gibbs, J. E. Evetts and J. A. Leake: J. Mater. Sci. 18 (1983) 278-288.

26) H. S. Chen, A. Inoue and T. Masumoto: J. Mater. Sci. 20 (1985) $2417-$ 2438.

27) A. Inoue, T. Masumoto and H. S. Chen: J. Mater. Sci. 20 (1985) 40574068 .

28) A. Inoue, T. Masumoto and H. S. Chen: J. Non-Cryst. Solids 83 (1986) 297-316.

29) O. Haruyama, M. Kohda, N. Nishiyama and T. Egami: J. Phys. Conf. Ser. 144 (2009) 012050.

30) O. Haruyama, J. Kasai and T. Egami: J. Non-Cryst. Solids to be submitted.

31) A. J. Kovacs: Adv. Polym. Sci. 3 (1963) 394.

32) O. Haruyama, K. Yoshikawa, Y. Yamasaki and Y. Yokoyama: Acta Mater. to be submitted.

33) L.-M. Wang, R. Liu and W. H. Wang: J. Chem. Phys. 128 (2008) 164503.

34) P. A. Duine, J. Sietsma and A. van den Beukel: Acta Metall. Mater. 40 (1992) 743-751. 
35) J. S. Langer: Phys. Rev. E 77 (2008) 021502.

36) T. Egami, K. Maeda and V. Vitek: Philos. Mag. A 41 (1980) 883-901.

37) T. Egami, K. Maeda, D. Srolovitz and V. Vitek: J. Phys. Colloq. 41 (1980) C8-272-274.

38) D. Srolovitz, T. Egami and V. Vitek: Phys. Rev. B 24 (1981) 69366944.

39) T. Egami, S. J. Poon, Z. Zhang and V. Kepens: Phys. Rev. B 76 (2007)
024203.

40) K. Kim and S. Saito: J. Chem. Phys. 133 (2010) 044511.

41) D. Kivelson, S. A. Kivelson, X. Zhao, Z. Nussinov and G. Tarjus: Physica A 219 (1995) 27-38.

42) C. A. Angell: Science 267 (1995) 1924-1935.

43) A. J. Kovacs, J. J. Aklonis, J. M. Hutchinson and A. R. Ramos: J. Polymer Sci. B 17 (1979) 1097-1162. 